\title{
SURVEY OF CORRELATED FIR, HI, CO, AND \\ RADIO-CONTINUUM EMISSION FEATURES IN THE \\ MULTI-PHASE MILKY WAY
}

\author{
W.F. WALL ${ }^{1,2,3}$ AND W.H. WALLER ${ }^{3,4}$ \\ ${ }^{1}$ INAOE, México \\ ${ }^{2} N A S A / G S F C, U S A$ \\ ${ }^{3}$ StarStuff Incorporated \\ ${ }^{4}$ Hughes STX, NASA/GSFC USA
}

\section{Introduction}

The interstellar medium (ISM) is rich with structure on varying size scales, reflecting its diverse energetics and dynamics. A step toward understanding this structure is to enhance the visibility of the structure on finer size scales. We present maps of a section of the Galactic plane filtered with the Median Normalized Spatial Filter (MNSF, a median smoothed map is divided into the original map), which emphasizes higher latitude emission relative to that of the plane itself (see also Waller et al., this volume, p. 194). The maps also illustrate the spatial correlations between the interstellar dust and the various gas phases: ionized, atomic, and molecular. The dust is represented by the IR emission maps of the IRAS survey, the ionized gas by the radio continuum maps at $1.4 \mathrm{GHz}$ (Reich 1978, northern sky) and $2.3 \mathrm{GHz}$ (Jonas \& Baart 1995, southern sky), the atomic gas by surveys of the HI $21 \mathrm{~cm}$ line emission (Heiles \& Habing 1974, Weaver \& Williams 1973, Cleary et al.1979, Kerr et al. 1986), and the molecular gas by surveys of the CO J $=1 \rightarrow 0$ line emission (Dame et al. 1987).

\section{Processing}

A rectangular window $15^{\circ}$ wide in longitude and 0.5 high in latitude was used in the spatial filtering. The MNSF was used for all the maps, except for the lower signal-to-noise $\mathrm{CO}$ maps, in which unsharp masking (i.e., 
smoothed map subtracted from the original map) was used. Maps of the dust-gas correlations were made by running a 2.5 -square box over the filtered IRAS and gas emission maps and, using the Spearman test, zeroing all positions in the IRAS map with probabilities of correlation (between gas and dust emission intensities) less than 0.9. The maps of correlated dust emission features were color-coded: red for correlation with the radio continuum, green for the HI line, blue for the CO line. The three color-coded maps were then superposed to form a true-color image.

\section{Results}

The resultant fine-scale maps are shown in Figure 1. Comparison of the top and bottom panels of Figure 1 show that the fine-scale structure in the dust emission at $100 \mu \mathrm{m}$ is in overall agreement with that of the gas. This is expected since the dust pervades most gas phases. The disagreements that do exist are sometimes attributable to the different way in which the $\mathrm{CO}$ map was filtered; the unsharp masking does not emphasize the $\mathrm{CO}$ emission away from the plane, as does the MNSF processing.

Clearly visible in the middle panel is a large number of FIR features that are correlated with the atomic phase. Analysis of positions within $11^{\circ}$ of the Galactic plane for all longitudes shows that $\sim 70 \%$ of the FIR structures are correlated with structured HI emission. The preponderance of atomic counterparts to the FIR structures evident away from the midplane indicates energetics that are sufficient to re-organize the ISM without ionizing it. Also, it should be mentioned that many of the correlated FIRHI features away from the mid-plane may also correlate with the molecular gas, which can be tested when a higher signal-to-noise ${ }^{12} \mathrm{CO} \mathrm{J}=1 \rightarrow 0$ map becomes available.

\section{Acknowledgements}

This research was supported in part by a NASA ADP contract to StarStuff Incorporated and by a CONACyT Grant to INAOE.

\section{References}

Cleary, M. N., Heiles, C., \& Haslam, C. G. T. 1979, Astron.Astrophys.Suppl., 36, 95

Dame, T. M., Ungerechts, H., Cohen, R. S., de Geus, E. J., Grenier, I. A., May, J., Murphy, D. C., Nyman, L.- $\AA .$, \& Thaddeus, P. 1987, Astrophys.J., 322, 706

Heiles, C. \& Habing, H. J. 1974, Astron.Astrophys.Suppl., 14, 1

Jonas, J.L. \& Baart, E. E. 1995, Astrophys.Sp.Sc., 230, 351

Kerr, F. J., Bowers, P. F., Jackson, P. D., \& Kerr, M. 1986, Astron.Astrophys.Suppl., 66,373

Reich, W. 1978, Astron.Astrophys., 64, 407

Weaver, H. \& Williams, W. 1973, Astron.Astrophys.Suppl., 8, 1 


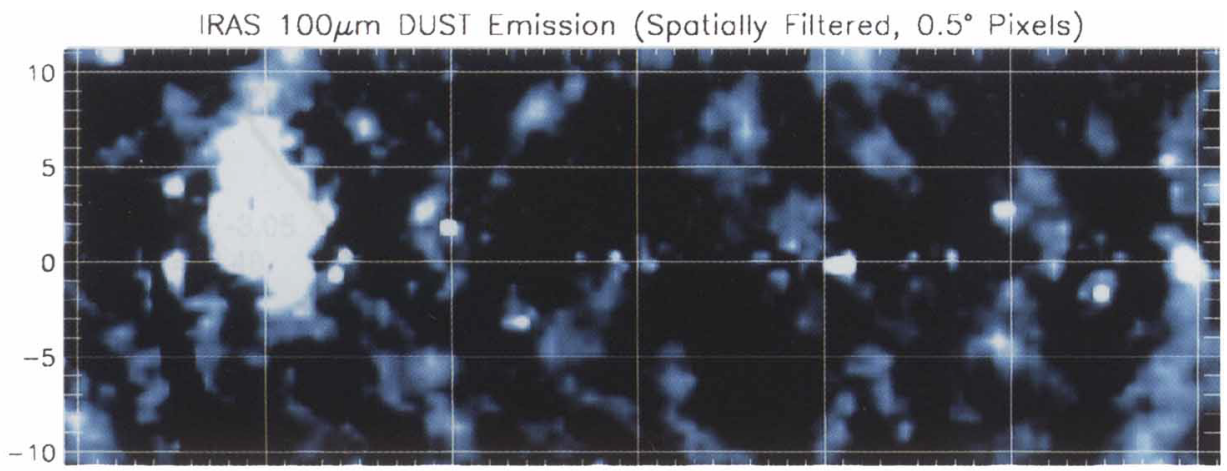

IRAS $100 \mu \mathrm{m}$ Emission Correlations, $(1.4 \mathrm{GHz}=$ Red, $\mathrm{HI}=$ Green, $\mathrm{CO}=\mathrm{Blue})$

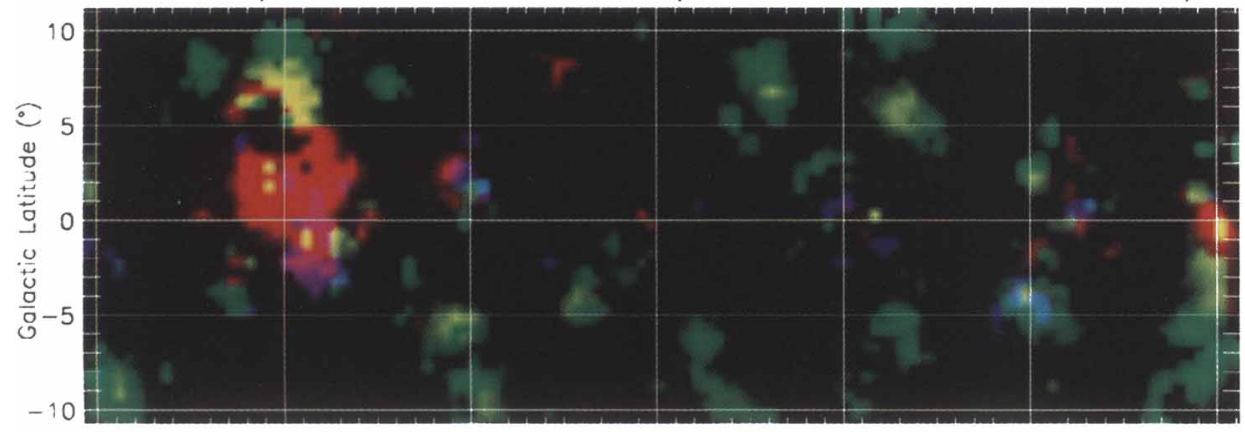

Spatially Filtered GAS Emission, (1.4GHz=Red, $\mathrm{HI}=$ Green, $\mathrm{CO}=\mathrm{Blue}$ )

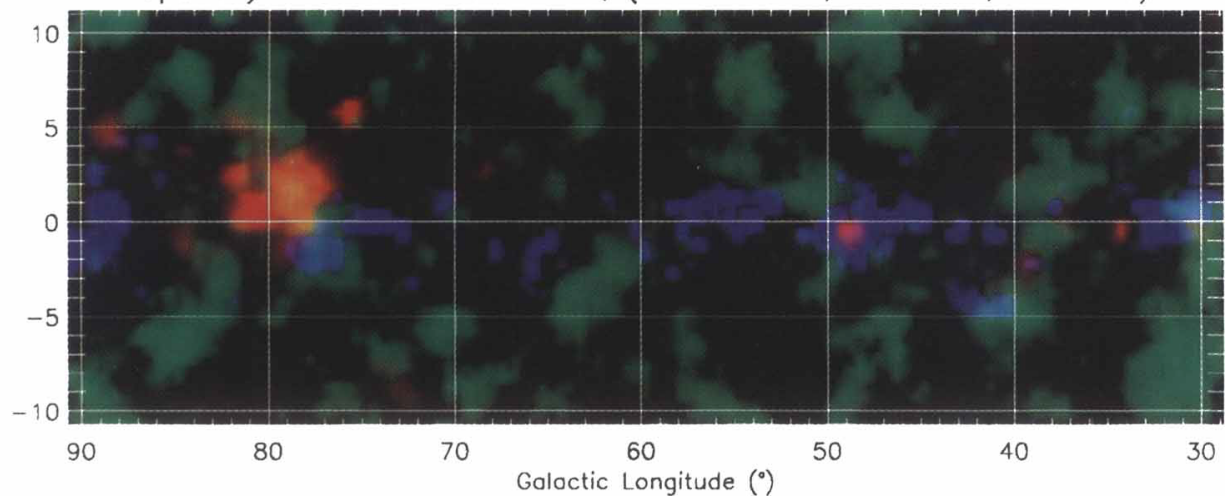

Figure 1. Maps of fine-scale structure of a section the Galactic plane are shown above. The top panel shows the dust emission at $100 \mu \mathrm{m}$ and the bottom panel shows the corresponding map of gas emission. The middle panels represent correlations of the $100 \mu \mathrm{m}$ emission with those of the gas components. 